\title{
Was ist kaputt: Meniskus, Bänder oder Knorpel?
}

\author{
Knieverletzungen gehören zu den häufigsten Komplikationen beim Sport. Vorrangig betrof- \\ fen sind Skiläufer und Fußballspieler. Um das Ausmaß und die betroffenen Gelenkstrukturen \\ zu erfassen, muss zunächst eine genaue Anamnese erhoben und eine sorgfältige klinische \\ Untersuchung durchgeführt werden, bevor die bildgebende Diagnostik zum Einsatz kommt.
}

\begin{abstract}
Die Anamnese und der klinische Befund geben wichtige Hinweise darauf, ob ein Meniskus-, Band- oder Knorpeloder eine Kombinationsverletzung vorliegt. Eine Knieverdrehung spricht eher
\end{abstract}

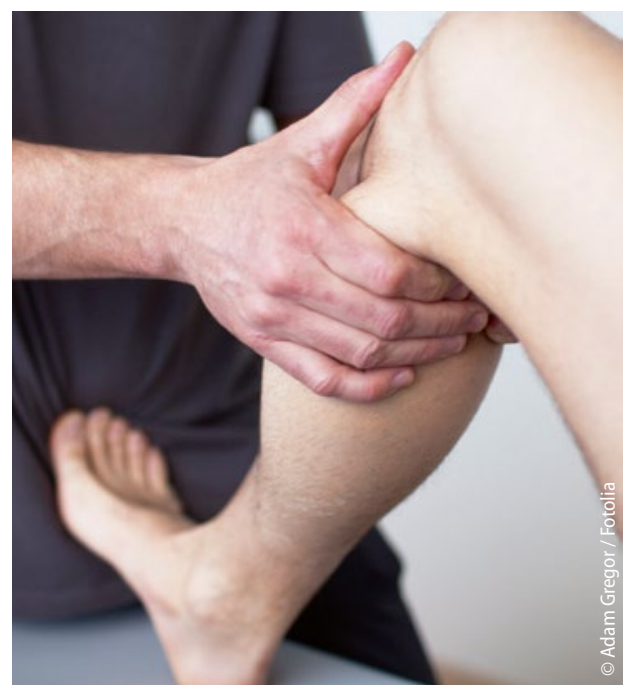

für einen Meniskusschaden, eine Torsionsverletzung bzw. ein Varus- oder Valgusstress deutet dagegen eher auf eine Bandläsion hin, und bei einer Stauchung oder einem Anprall sollte man primär an eine Knorpelverletzung denken.

\section{Genauer Gelenkstatus}

Bei der klinischen Untersuchung sollte auf das Vorliegen eines Gelenkergusses geachtet werden. Die „tanzende Patella“ ist dafür beweisend. Gibt der Patient Schmerzen beim Verschieben der Patella an, so spricht dies für eine Retropatellararthrose. Der Nachweis eines Meniskusschadens gelingt mittels Rotationsbewegungen, wobei ein Schmerz am inneren Gelenkspalt bei Außenrotation für eine Innenmeniskusläsion, ein Schmerz am äußeren Gelenkspalt bei der Innenrotation für einen Außenmeniskusschaden spricht. Bei Kreuzbandverletzungen findet sich das Schubladen- phänomen. überdies sollte die mediale und laterale Aufklappbarkeit des Gelenks geprüft werden, um die Stabilität der Seitenbänder beurteilen zu können.

\section{Sofort-Therapie nach der PECH-Regel}

Die Soforttherapie einer Kniegelenksverletzung, auch dann, wenn eine operative Therapie geplant ist, sollte nach der PECH-Regel erfolgen. Dabei stehen

$P$ für Pause, also mit dem Sport aufhören

E für Eis, also sofortige Kühlung

C für Compression, also einen Druckverband mit mäßiger Spannung

H für Hochlagerung, wodurch die Blutzufuhr in das geschädigte $\mathrm{Ge}$ webe gedrosselt und der Abtransport der Gewebeflüssigkeit erleichtert wird.

Dr. med. Peter Stiefelhagen

- 46. Deutscher Sportärztekongress, Frankfurt a. M. 11.- 12.9.2015

\section{Quo vadis Sportmedizin?}

\author{
Welches Profil braucht die deutsche Sportmedizin, um ihre Rolle in Prävention und Therapie festigen \\ und auszubauen zu können? Wie sollte sie sich in Praxis und Wissenschaft weiterentwickeln?
}

\begin{abstract}
_ Dies sind aktuelle Fragen, die auch beim diesjährigen Sportärztekongress diskutiert wurden. Mit Prof. Michael Kjaer aus Kopenhagen hatte man einen international anerkannten Kenner der sportmedizinischen Szene eingeladen, der aus Sicht eines nicht in die nationalen Strukturen eingebundenen Externen und zugleich kritischen Experten seine Vorstellungen im Rahmen eines Plenarvortrags darlegen konnte.

Ob man einen Facharzt für Sportmedizin einführen soll, diese Frage wird kon-
\end{abstract}

trovers diskutiert, „Da gibt es ein Pro und ein Contra“, so Kjaer. Ein solcher Facharzt werte zwar das Image des Faches auf andererseits aber habe dieser Facharzt in den verschiedenen Fachdisziplinen, die die Sportmedizin als Querschnittsfach berühre, doch nur eine begrenzte Kompetenz. Wichtig allerdings sei, dass die Deutungshoheit über bestimmte Behandlungsverfahren nicht nur den einzelnen Disziplinen überlassen bleibe, sondern auch von sportmedizinischer Seite beurteilt werden müsse. Dazu gehören Fragen wie z. B., ob zu viel Menisken operiert oder zu viel Koronarinterventionen durchgeführt werden, also ob die Langzeitergebnisse solcher Eingriffe die großzügige Indikationsstellung rechtfertigen. „Auch muss die Frage erlaubt sein, ob Statine durch ihre Nebenwirkungen am Muskel eine Inaktivität provozieren, welche die präventive Wirkung des Medikaments vielleicht zunichte macht“, so Kjaer. Bei all diesen Fragestellungen müsse die Sportmedizin sich stärker einbringen. Nur so könne die universitäre Sportmedizin stark werden. - sti 